\title{
Computational Experiment for Stability Analysis of Difference Schemes with Nonlocal Conditions
}

\author{
Mifodijus SAPAGOVAS ${ }^{1}$, Regimantas ČIUPAILA $^{2}$, \\ Živilè JOKŠIENË ${ }^{3}$, Tadas MEŠKAUSKAS ${ }^{4}$ \\ ${ }^{1}$ Vilnius University Institute of Mathematics and Informatics \\ Akademijos 4, LT-08663 Vilnius, Lithuania \\ ${ }^{2}$ Vilnius Gediminas Technical University \\ Sauletekio al. 11, LT-10223 Vilnius, Lithuania \\ ${ }^{3}$ Lithuanian University of Health Sciences, Vytautas Magnus University \\ Eiveniu 4, LT-50009 Kaunas, Lithuania \\ ${ }^{4}$ Vilnius University, Faculty of Mathematics and Informatics \\ Naugarduko 24, LT-03225 Vilnius, Lithuania \\ e-mail: mifodijus.sapagovas@mii.vu.lt,regimantas.ciupaila@vgtu.lt,zivile.je@gmail.com, \\ tadas.meskauskas@mif.vu.lt
}

Received: December 2011; accepted: February 2013

\begin{abstract}
Based on an example, we describe how outcomes of computational experiment can be employed for study of stability of numerical algorithm, provided that related theoretical propositions are not proven yet. More precisely, we propose a systematic and generalized methodology, how to investigate the influence of the weight functions $\alpha(x)$ and $\beta(x)$, present in the integral boundary conditions, on the stability of difference schemes, for some class of parabolic equations. The ground of the methodology is the investigation of the spectrum of a matrix, defining the transition to the upper layer of the difference scheme. Spectral structure of this matrix is analysed by both analytic method and computational experiment.
\end{abstract}

Keywords: parabolic equation, nonlocal integral condition, difference scheme, stability, computational experiment.

\section{Introduction and Problem Statement}

It is a common practice in research, related to numerical analysis, to employ a computational experiment, alongside of a theoretical study. Such a practice started a few decades ago, with advent of powerful computers. Nowadays, even more mighty environments (clusters, grids, supercomputers) are available for scientific computing, making computational research possible for truly demanding (two-dimensional, three-dimensional, etc.) problems.

The distinguishing feature of computational research is that one performs computations (on a computer or in some computational environment), aiming to reveal yet unknown patterns, laws, trying to suggest conjectures - instead of seeking to illustrate,

\footnotetext{
${ }^{*}$ Corresponding author.
} 
verify or explain already proven theoretical propositions. Years from now, computational research has proved useful for studying phenomena and processes in physics, described by differential models, see, for example the work Samarskii (1979). These days, computational experiment is part of research in many fields of informatics. In Medvedev and Dzemyda (2006), Medvedev et al. (2011) the computational experiment was used for data analysis aiming to make conclusions about the effectiveness of the numerical methods.

In this paper, we demonstrate employment of computational experiment in a particular branch (which is still under significant development) of numerical analysis - numerical algorithms for differential equations, subject to nonlocal boundary conditions. As an example of such mathematical model, one-dimensional (in space variable) parabolic equation, subject to one or two integral conditions (instead of frequently used classical boundary conditions) is dealt with often.

Our choice of this particular problem has been motivated by different applications as well as by interesting and not finally answered questions about the stability of difference schemes. Cannon (1963) investigated the heat equation, subject to the specification of energy. Day (1982) applied nonlocal conditions when dealing with problems of the linear thermoelasticity theory. Yin (2004) considered a generalized model for theory of iondiffusion in channels. The thermostat problem with nonlinear nonlocal conditions was investigated in the article Kalna and McKee (2004). A lot of differential equations of various types with nonlocal conditions can be found in books of Schuegerl (1987) and Nakhushev (1995).

In this work, we are going to focus on the parabolic equation

$$
\frac{\partial u}{\partial t}=\frac{\partial^{2} u}{\partial x^{2}}+f(x, t), \quad x \in(0,1), t \in(0, T],
$$

subject to two nonlocal integral conditions

$$
\begin{aligned}
& u(0, t)=\gamma_{1} \int_{0}^{1} \alpha(x) u(x, t) d x+\mu_{1}(t), \\
& u(1, t)=\gamma_{2} \int_{0}^{1} \beta(x) u(x, t) d x+\mu_{2}(t),
\end{aligned}
$$

instead of classical boundary conditions. The initial values are defined in a standard way:

$$
u(x, 0)=\varphi(x) .
$$

Here $u(x, t)$ denotes an unknown function (a solution of the above differential problem), $\gamma_{1}$ and $\gamma_{2}$ are given parameters (real numbers), $f(x, t), \alpha(x), \beta(x), \mu_{1}(t), \mu_{2}(t)$ and $\varphi(x)$ - given functions. Note that under the special case of $\gamma_{1}=\gamma_{2}=0$ nonlocal integral conditions (2), (3) become familiar (classical) boundary conditions.

Ekolin (1991) analysed the stability of a difference scheme for a parabolic equation subject to integral condition, by applying maximum principle. Note though, that in this 
paper, proof of stability assumes essential boundedness of the quantities $\left|\gamma_{1} \alpha(x)\right|$ and $\left|\gamma_{2} \beta(x)\right|$, in other words - the nonlocal conditions (2), (3) must be rather close to classical ones. Čiegis et al. (2002), and Čiegis and Tumanova (2010) got more general results, concerning stability (of the difference schemes) conditions. Wider reviews of such kind problems are provided in the papers of Dehghan (2005, 2007), Ivanauskas et al. (2009).

The method, based on investigation of the spectral structure of difference operators with nonlocal conditions, for examination of the stability of the difference schemes for parabolic equations (with nonlocal conditions) has been widely used during the last decade (Gulin et al., 2006; Ivanauskas et al., 2009; Sapagovas, 2008). Other works, employing study of the spectral structure of difference operators (with nonlocal conditions), are the papers of Čiupaila et al. (2004), Jesevičiūtè and Sapagovas (2008), Sapagovas and Štikonas (2005). Differently from differential or difference operators with classical boundary conditions, the spectral structure of the operators with nonlocal conditions can be rather complicated (Sapagovas and Štikonas, 2005). In many cases the spectral structure was successfully investigated by using both analytic and numerical methods in one study (Skučaite et al., 2010; Štikonas, 2011).

In the paper of Dehghan (2005) the problem (1)-(4) was solved using various difference methods, for example, Crandall's formula. Applying this algorithm the differential equation (1) is replaced by the difference equation

$$
\frac{u_{i}^{n+1}-u_{i}^{n}}{\tau}=\left(\frac{1}{2}+\frac{1}{12 s}\right) \Lambda u_{i}^{n}+\left(\frac{1}{2}-\frac{1}{12 s}\right) \Lambda u_{i}^{n+1}+g_{i}^{n},
$$

where

$$
\Lambda u_{i}^{n}=\frac{u_{i-1}^{n}-2 u_{i}^{n}+u_{i+1}^{n}}{h^{2}}, \quad s=\frac{\tau}{h^{2}},
$$

$\tau=T / M, h=1 / N$, and $g_{i}^{n}$ approximates the function $f(x, t)$ in the point $\left(x_{i}, t_{n}\right)$. Integrals in (2) and (3) are replaced by Simpson's rule. Such difference scheme (with particularly chosen $g_{i}^{n}$ ) approximates the differential problem (1)-(4) with approximation error $O\left(h^{4}+\tau^{2}\right)$. In the work of Dehghan (2005) it was stated that the discussed scheme is stable. In the paper of Martin-Vaquero and Vigo-Aguiar (2009a) Crandall's formula was modified, some inaccuracies were corrected. Three testing problems were solved in the same paper of Martin-Vaquero and Vigo-Aguiar (2009a), proving, according to the authors of that paper, high accuracy of the method discussed.

In the article of Martin-Vaquero and Vigo-Aguiar (2009b) another family of difference methods was investigated. Therein $\tau=h$ and $p$-level $(p=2,3,4,5)$ difference schemes were proposed. As a special case, an efficient scheme with accuracy $O\left(h^{4}+\tau^{4}\right)$ was discussed (in general, approximation errors of the analysed algorithms were shown to be $O\left(h^{p-1}+\tau^{p-1}\right)$ ). If $p=2$, the method under consideration presents a well-known implicit scheme, where (1) is replaced by the difference equation

$$
\frac{u_{i}^{n+1}-u_{i}^{n}}{\tau}=\Lambda u_{i}^{n+1}+f_{i}^{n+1}, \quad \tau=h .
$$


When $p=2$, the stability of the scheme is proven, while the stability in the cases $p=3,4,5$ is claimed to follow analogously.

Another important (and interesting) question is the impact of the weight functions $\alpha(x)$ and $\beta(x)$ (appearing in the nonlocal conditions (2) and (3)) on the stability of a difference scheme. None of the papers of Dehghan (2005), Martin-Vaquero and Vigo-Aguiar (2009a, 2009b) address this question.

The aim of our paper is to investigate using the computational experiment the influence of the nonlocal conditions (2) and (3) on the stability of difference schemes, based on (5).

From computational point of view, to achieve this goal we were presented with demanding (in terms of CPU time) task. We have implemented a tool for computing all eigenvalues of a square matrix (of order, which in computational experiments exceeds 100). This tool produces output pretty fast (even on a personal computer) for single combination of parameters $\gamma_{1}$ and $\gamma_{2}$ (and with all other parameters and functions of mathematical model and algorithm fixed). However, to obtain a full and detailed picture of spectral structure (of a difference operator) dependence on the nonlocal conditions (2) and (3), one has to run computations in two-dimensional $\left(\gamma_{1}, \gamma_{2}\right)$ parameters space. Moreover, computational experiment must be repeated with many different combinations of the weight functions $\alpha(x)$ and $\beta(x)$. Therefore, we were in demand for much more efficient (compared to a personal computer) computing platform. Two such platforms were available for us: (1) BalticGrid grid environment and (2) supercomputer deployed in Digital Science and Computing Centre of Vilnius University, Faculty of Mathematics and Informatics.

Beside the tool for computational spectral analysis, a differential solver, based on the difference scheme, has been implemented, too.

All tools were written in C programming language, calling subroutines of GNU Scientific Library and adapted for BalticGrid and supercomputer. Compared to C, high-level programming languages (e.g., under open source license: Python, Octave; commercially licensed: Mathematica, MATLAB, Maple) would allow a simpler implementation, but some issues (speed, portability, availability) may also arise, especially when compiling and executing code in such platforms as grid and supercomputer. Note though, that alternative implementation (in some high-level programming language) can be employed for testing of developed software.

We state the main result of this paper as follows. The stability of the difference scheme, approximating the differential problem (1)-(4) essentially depends on the weight functions $\alpha(x)$ and $\beta(x)$, and not on their absolute values, but on their integral characteristics. This dependence can be determined by the spectral structure of transition matrix of two-level difference scheme. The spectral structure is studied by combining analytic methods with computational experiment.

\section{Investigation of Difference Schemes with Nonlocal Conditions}

In this section we shall consider methodology, how the stability of schemes with nonlocal conditions can be investigated. The main ideas of the methodology are provided 
in the works of Gulin et al. (2006), Jachimavičienè et al. (2009), Sapagovas (2008).

Let us replace the problem (1)-(4) by the following weighted (with weight $\sigma$ ) difference scheme

$$
\begin{aligned}
& \frac{u_{i}^{n+1}-u_{i}^{n}}{\tau}=\sigma \Lambda u_{i}^{n+1}+(1-\sigma) \Lambda u_{i}^{n}+g_{i}^{n}, \quad i=1,2, \ldots, N-1, \\
& u_{0}^{n+1}=\gamma_{1}\left(\alpha, u^{n+1}\right)+\mu_{1}^{n+1} \\
& u_{N}^{n+1}=\gamma_{2}\left(\beta, u^{n+1}\right)+\mu_{2}^{n+1} \\
& u_{i}^{0}=\varphi_{i}, \quad i=0,1, \ldots, N .
\end{aligned}
$$

Here

$$
\left(\alpha, u^{n+1}\right)=\frac{h}{3}\left(\alpha_{0} u_{0}^{n+1}+\alpha_{N} u_{N}^{n+1}+4 \sum_{i=1}^{N / 2} \alpha_{2 i-1} u_{2 i-1}^{n+1}+2 \sum_{i=1}^{N / 2-1} \alpha_{2 i} u_{2 i}^{n+1}\right)
$$

i.e., $\left(\alpha, u^{n+1}\right)$ is the expression of the Simpson's rule. The value $\left(\beta, u^{n+1}\right)$ is defined analogously. The number $N$ is even, $g_{i}^{n}$ is some approximation of the function $f(x, t)$. For example,

(1) when $\sigma=1, g_{i}^{n+1}=f_{i}^{n+1}$, then (7) turns into the implicit equation (6), approximation error is $O\left(h^{2}+\tau\right)$;

(2) when $\sigma=1 / 2, g_{i}^{n+1}=f_{i}^{n+1 / 2}$, we get symmetric Crank-Nicolson scheme, approximation error is $O\left(h^{2}+\tau^{2}\right)$;

(3) when $\sigma=\frac{1}{2}-\frac{h^{2}}{12 \tau}, g_{i}^{n+1}=f_{i}^{n+1 / 2}+\frac{h^{2}}{12} \Lambda f_{i}^{n+1 / 2}$, (7) coincides with the Crandall's formula (5), approximation error $O\left(h^{4}+\tau^{2}\right)$ - the so called scheme of increased order of accuracy (Samarskii, 2001).

Let us take the difference scheme (7)-(9) written down in a standard way on the $(n+1)$ th layer (when $\left.t=t^{n+1}\right)$

$$
u^{n+1}=S u^{n}+\bar{f}^{n},
$$

here $u^{n}=\left(u_{1}^{n}, u_{2}^{n}, \ldots, u_{N-1}^{n}\right)$ and $S$ - square matrix of order $N-1$. From the conditions (8) and (9) we express $u_{0}^{n+1}$ and $u_{N}^{n+1}$ through other unknowns of the same layer, to get the system of two equations

$$
\begin{aligned}
& u_{0}^{n+1}=\frac{1}{D}\left(\sum_{i=1}^{N-1} \widetilde{\alpha}_{i} u_{i}^{n+1}+\widetilde{\mu}_{1}^{n+1}\right), \\
& u_{N}^{n+1}=\frac{1}{D}\left(\sum_{i=1}^{N-1} \widetilde{\beta}_{i} u_{i}^{n+1}+\widetilde{\mu}_{2}^{n+1}\right) .
\end{aligned}
$$


Here $\widetilde{\alpha}_{i}, \widetilde{\beta}_{i}, \widetilde{\mu}_{1}^{n+1}, \widetilde{\mu}_{2}^{n+1}$ are some known expressions (that will not be employed further in the paper), depending on the values $\gamma_{1}, \gamma_{2}, \alpha_{i}, \beta_{i}, \mu_{1}^{n+1}, \mu_{2}^{n+1}$, and

$$
D=\left|\begin{array}{cc}
1-\frac{\gamma_{1} \alpha_{0} h}{3} & -\frac{\gamma_{1} \alpha_{N} h}{3} \\
-\frac{\gamma_{2} \beta_{0} h}{3} & 1-\frac{\gamma_{2} \beta_{N} h}{3}
\end{array}\right| .
$$

The following Lemma is stated without proof (which is straightforward).

\section{Lemma 1. If}

$$
\left|\gamma_{1} \alpha_{i}\right| \leqslant M, \quad\left|\gamma_{2} \beta_{i}\right| \leqslant M
$$

and $M h<3 / 2$ (i.e., $h$ is sufficiently small), then $D>0$.

We conclude from Lemma 1, that the expressions (12) and (13) are well-defined, if the grid step $h$ is sufficiently small.

By substituting the expressions of $u_{0}^{n+1}$ and $u_{N}^{n+1}$ into the difference equation (7) (in the cases of $i=1$ and $i=N-1$, respectively), we get the equality (11), in which

$$
S=(I+\tau \sigma A)^{-1}(I-\tau(1-\sigma) A)
$$

where $I$ is the identity matrix, and square matrix $A$ of order $N-1$ is defined as follows:

$$
A=\frac{1}{h^{2}}\left(\begin{array}{rrrrrr}
2-a_{1} & -1-a_{2} & -a_{3} & \cdots & -a_{N-2} & -a_{N-1} \\
-1 & 2 & -1 & \ddots & 0 & 0 \\
0 & -1 & 2 & \ddots & 0 & 0 \\
\vdots & \ddots & \ddots & \ddots & \ddots & \vdots \\
0 & 0 & 0 & \ddots & 2 & -1 \\
-b_{1} & -b_{2} & -b_{3} & \cdots & -1-b_{N-2} & 2-b_{N-1}
\end{array}\right),
$$

The matrix $A$ differs from a tridiagonal matrix only by first and last rows, which are full. An eigenvalue problem for this matrix could be presented as an eigenvalue problem for a difference operator with nonlocal conditions. 
Lemma 2. An eigenvalue problem of the matrix $A$ (of order $N-1$ ) is equivalent to an eigenvalue problem of the following difference operator (with nonlocal conditions):

$$
\begin{aligned}
& \Lambda u_{i}+\lambda u_{i}=0, \quad i=1,2, \ldots, N-1, \\
& u_{0}=\gamma_{1}(\alpha, u), \\
& u_{N}=\gamma_{2}(\beta, u) .
\end{aligned}
$$

Lemma 2 has been proven in the paper of Sapagovas (2008), having assumed that integrals in the conditions (2) and (3) are approximated by the trapezoid rule. In the case of Simpson's rule proof is analogous.

The study of Sapagovas (2008) also contains proofs of the following statements, which we shall formulate as Theorems 1 and 2. We shall employ them in Section 3.

Theorem 1 (Sapagovas, 2008). If all eigenvalues $\lambda_{i}(A)$ (of the matrix $A$ ) are real and positive or meet the condition $\operatorname{Re} \lambda_{i}(A)>0$, then

(1) if $1 / 2 \leqslant \sigma \leqslant 1$, then $\rho(S)=\max _{1 \leqslant i \leqslant N-1}\left|\lambda_{i}(A)\right|<1$;

(2) if $0 \leqslant \sigma<1 / 2$, then $\rho(S)<1$ with some additional constraint on $\tau / h^{2}$.

The condition $\rho(S)<1$ assures the stability of the difference scheme. That is, the criterion $\operatorname{Re} \lambda_{i}(A)>0$ presents the sufficient condition for the stability of the difference scheme (11). This condition could be weakened to the condition $\operatorname{Re} \lambda_{i}(A) \geqslant 0$. But if at least one eigenvalue (of the matrix $A$ ) is such that $\operatorname{Re} \lambda_{i}(A)<0$, the scheme becomes unstable. We have come to this conclusions by performing sizeable computational experiment.

One of the main objectives (investigating the stability of the scheme (11)) was to obtain simply verifiable condition, equivalent to the existence of the eigenvalue $\lambda=0$ of the matrix $A$ (or, in other words - of the difference operator (17)-(19)).

Theorem 2 (Sapagovas, 2008). The number $\lambda=0$ is the eigenvalue of the difference operator (17)-(19) if and only if the point $\left(\gamma_{1}, \gamma_{2}\right)$ rests on the characteristic curve

$$
\{(\alpha, x)(\beta, 1)-(\beta, x)(\alpha, 1)\} \gamma_{1} \gamma_{2}+\{(\alpha, 1)-(\alpha, x)\} \gamma_{1}+(\beta, x) \gamma_{2}-1=0 .
$$

In general, the curve (20) defines a hyperbola in the coordinate plane $\left(\gamma_{1}, \gamma_{2}\right)$. However, depending on values of $\alpha(x)$ and $\beta(x)$, it can degrade to a line, two lines or even be an empty set. If $\alpha(x) \geqslant 0$ and $\beta(x) \geqslant 0(\alpha(x) \not \equiv 0, \beta(x) \not \equiv 0)$, as $x \in(0,1)$, then the curve (20) can present a hyperbola (with asymptotes, parallel to the coordinates axes) or the line (if one branch of hyperbola digresses to infinity and another one becomes a line).

In Fig. 1 the characteristic curve (20), defining a hyperbola in the case of $\alpha(x)>0$, $\beta(x)>0($ as $x \in(0,1))$ and $(\alpha-\beta, x)>0$, is displayed.

Two branches of the hyperbola divide the whole coordinate plane $\left(\gamma_{1}, \gamma_{2}\right)$ into three unbounded domains (or two domains, if the characteristic curve presents a line). One part 


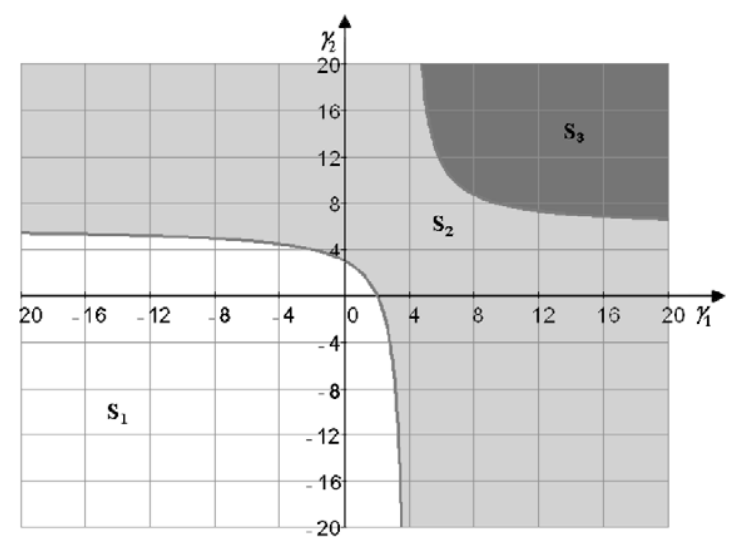

Fig. 1. The hyperbola (20) in the coordinate plane $\left(\gamma_{1}, \gamma_{2}\right)$, with $\alpha(x)=1, \beta(x)=x$.

of the coordinate plane, to which the point $(0,0)$ belongs, we mark as $S_{1}$, and the other two as $S_{2}$ and $S_{3}$ (see Fig. 1). When $\gamma_{1}=\gamma_{2}=0$, all $N-1$ eigenvalues of the problem (17)-(19) are real and positive. Therefore, in the vicinity of the point $\left(\gamma_{1}=0, \gamma_{2}=0\right)$ (that is, in some subset of the domain $S_{1}$ ) the entire spectrum of the matrix $A$ is positive too, hence the difference scheme (11) is stable here.

If, for any $\gamma_{1}, \gamma_{2}$, the matrix $A$ would not possess complex (with non-zero imaginary part) eigenvalues (note that $A$ is asymmetric, except of the case $\gamma_{1}=\gamma_{2}=0$ ), then in the entire domain $S_{1}$ the difference scheme would be stable. It is worth mentioning that in the domains $S_{2}$ and $S_{3}$ one or two negative eigenvalues usually exist. Numerical evidence suggests that in regions (of the coordinate plane $\left(\gamma_{1}, \gamma_{2}\right)$ ), where negative eigenvalues are present, the difference scheme is unstable.

However, the existence of complex eigenvalues can have essential impact on the stability (see Tests 1-4 in Section 3).

\section{Results of Computational Experiment}

We are going to perform four specific numerical tests, which have been dealt with in the papers of Martin-Vaquero and Vigo-Aguiar (2009a, 2009b).

Test 1. Let's deal with the problem (1)-(4) and take

$$
\alpha(x)=\beta(x)=x .
$$

The functions $f(x, t), \mu_{1}(t), \mu_{2}(t)$ and $\varphi(x)$ were chosen such that the exact solution of the problem would be

$$
u(x, t)=\left(\frac{x}{t+1}\right)^{2}
$$

for any values of $\gamma_{1}, \gamma_{2}$. 


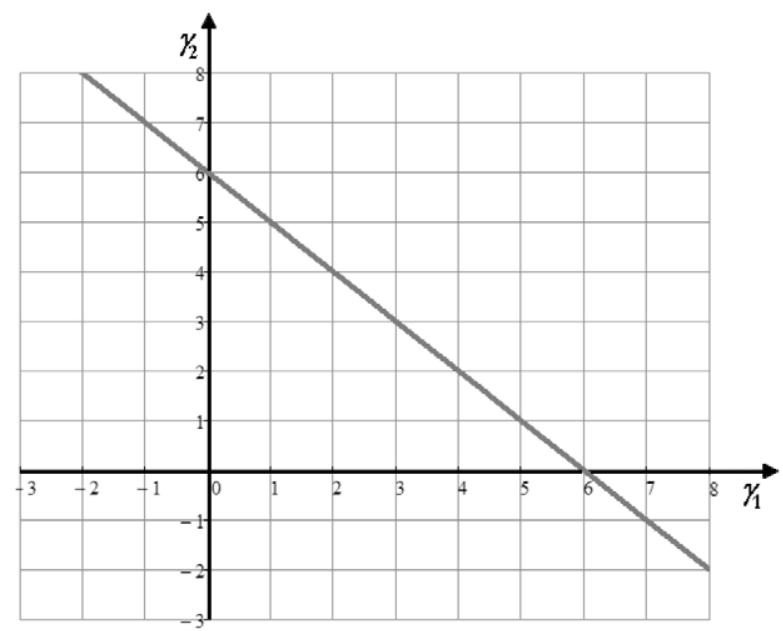

Fig. 2. The line (20) in the coordinate plane $\left(\gamma_{1}, \gamma_{2}\right)$, with $\alpha(x)=\beta(x)=x$.

The characteristic curve in this case is the line (see Fig. 2)

$$
\gamma_{1}+\gamma_{2}-6=0
$$

In Fig. 3 the properties of the spectrum of the matrix $A$ are presented.

The part of the plane $\left(\gamma_{1}, \gamma_{2}\right)$, where at least one negative or holding the property $\operatorname{Re} \lambda_{i}(A)<0$ eigenvalue exists, is displayed in the gray background. If the point $\left(\gamma_{1}, \gamma_{2}\right)$ depends to the gray background domain, then, with these values of $\gamma_{1}, \gamma_{2}$, the difference scheme (11) is unstable.

Analogously, in the region of white background all eigenvalues of the matrix $A$ are positive, and in the criss-crossed area of white background all eigenvalues possess the property $\operatorname{Re} \lambda_{i}(A)>0$ (some eigenvalues in this region are complex, with non-zero imaginary part). When the point $\left(\gamma_{1}, \gamma_{2}\right)$ depends to any of these two regions, the difference scheme (11) is stable.

This test with the parameters $\gamma_{1}=\gamma_{2}=1$ (we have marked this point in the right part of Fig. 3) has been dealt with numerically in the papers of Martin-Vaquero and VigoAguiar (2009a, 2009b) and the stability of the difference method has been observed. Our computer experiment with the same values $\gamma_{1}=\gamma_{2}=1$ affirms that the difference scheme (11) is indeed stable. Moreover, we have indicated values of $\gamma_{1}, \gamma_{2}$ such that the difference scheme is unstable (Fig. 3, region of gray background).

Test 2. Now, we examine the problem (1)-(4) with

$$
\alpha(x)=x, \quad \beta(x)=\cos x
$$

and the functions $f(x, t), \mu_{1}(t), \mu_{2}(t), \varphi(x)$ defined so, that the exact solution would be

$$
u(x, t)=e^{-(x+\sin t)} .
$$



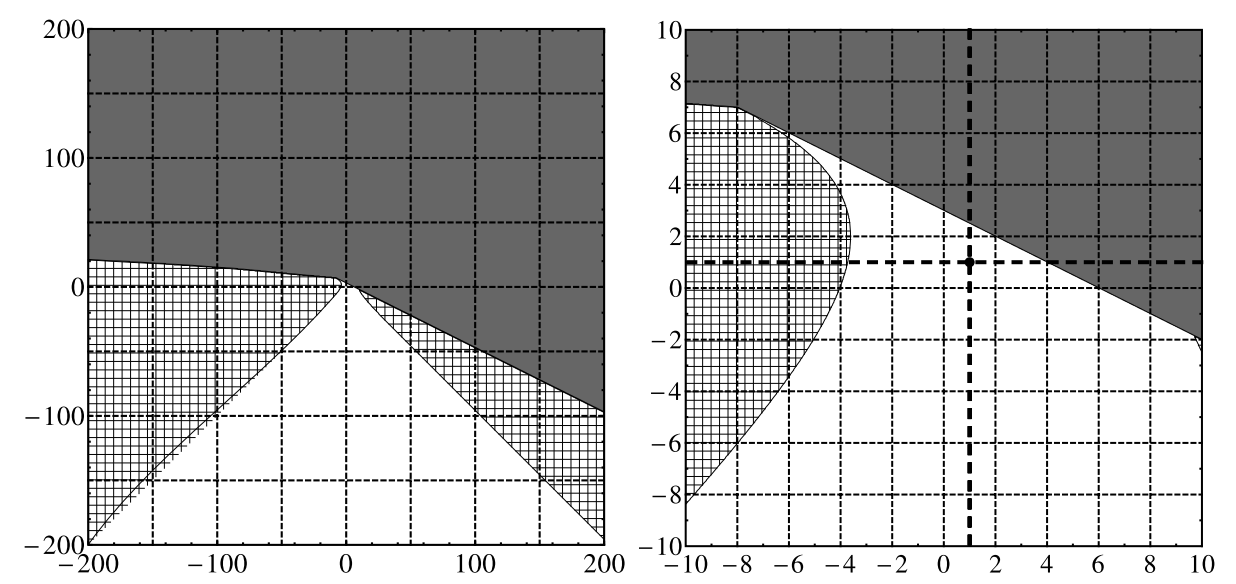

Fig. 3. Properties of the spectrum of the matrix $A$ in the plane $\left(\gamma_{1}, \gamma_{2}\right) . \alpha(x)=x, \beta(x)=x, N=200$, $h=0.005$. Figure on the right presents the zoom of the figure on the left.
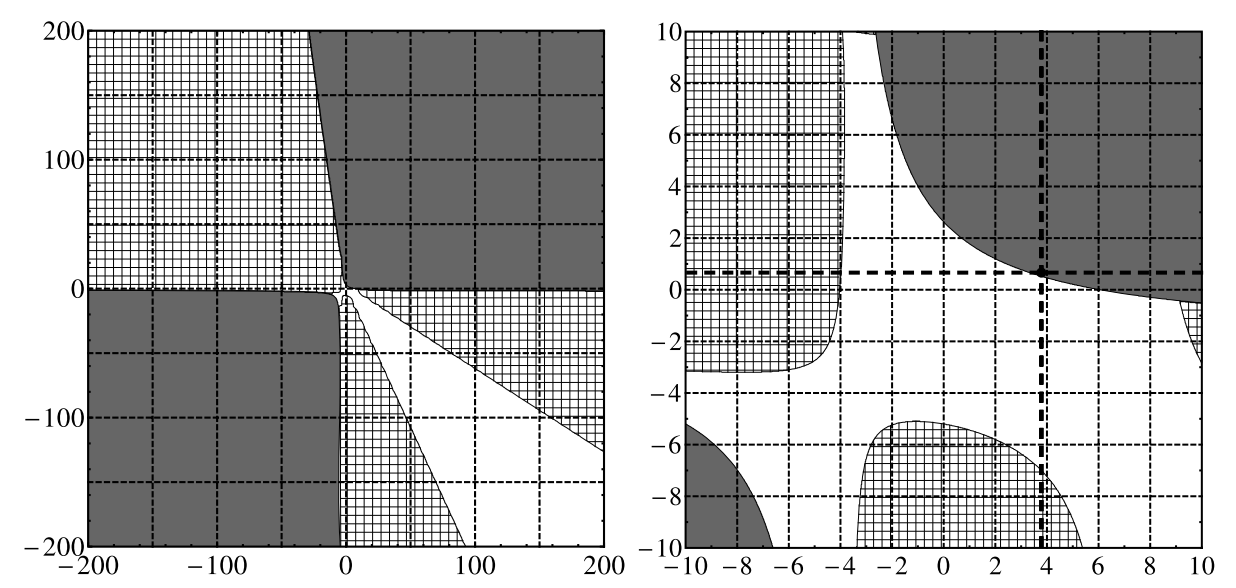

Fig. 4. Properties of the spectrum of the matrix $A$ in the plane $\left(\gamma_{1}, \gamma_{2}\right) . \alpha(x)=x, \beta(x)=\cos x, N=200$, $h=0.005$. Figure on the right presents the zoom of the figure on the left.

In the papers of Martin-Vaquero and Vigo-Aguiar (2009a, 2009b) this test has been solved numerically with

$$
\gamma_{1}=\frac{e}{e-2} \approx 3.78442, \quad \gamma_{2}=\frac{2}{e+\sin 1-\cos 1} \approx 0.662372
$$

We have marked this point in the right part of Fig. 4.

The authors of the last mentioned papers claim, that due to numerical results, obtained in the time interval $0 \leqslant t \leqslant 1$, the difference method is stable.

However, our results show, that situation concerning the stability of the difference scheme is much more complicated. 
In the case under consideration, the characteristic curve (20) presents the hyperbola (in the right part of Fig. 4 it coincides with the boundary of gray regions)

$$
0.090 \gamma_{1} \gamma_{2}+0.167 \gamma_{1}+0.382 \gamma_{2}-1=0
$$

Figure 4 employs graphical notations (regions with different background), matching with those, defined in Test 1. That is, in Fig. 4, same as it was in Fig. 3, regions of instability (of the difference scheme) appears in gray background.

The point $\left(\gamma_{1}=3.78442, \gamma_{2}=0.662372\right)$ is in the zone of instability, but quite close to the characteristic curve. With these values of $\gamma_{1}$ and $\gamma_{2}$ the matrix $A$ possesses one negative eigenvalue (approximately equal to minus one), all other eigenvalues are positive. Because there exists only one negative eigenvalue $\lambda_{1} \approx-1\left(\lambda_{1}=-0.999794\right.$, if $N=100 ; \lambda_{1}=-0.999998$, if $N=1000$ ), calculations based on unstable difference scheme in some bounded interval of time $t$ (for example, $0 \leqslant t \leqslant 1$ ) can look reliable enough (error of the solution grows as $e^{-\lambda_{1} t}, \lambda_{1}<0$ ). But this conclusion would be a wrong one. In the left part of Fig. 5 we see how error of the solution grows, depending on increasing time $t$. Even if the error is still small in the beginning, an exponential grow of the error is observed soon. Therefore we conclude that the difference scheme is actually unstable. It is worth mentioning, that in this case we witness analogous situation as it was described in the paper Lin et al. (1997) for two-dimensional parabolic equation, i.e., the error starts to grow unboundedly, as $t$ increases.

Also, we have computed the case $\gamma_{1}=-4, \gamma_{2}=8$, belonging to the stability region (see Fig. 4). Evolution of the error, displayed in the right part of Fig. 5, confirms the stability of the scheme.
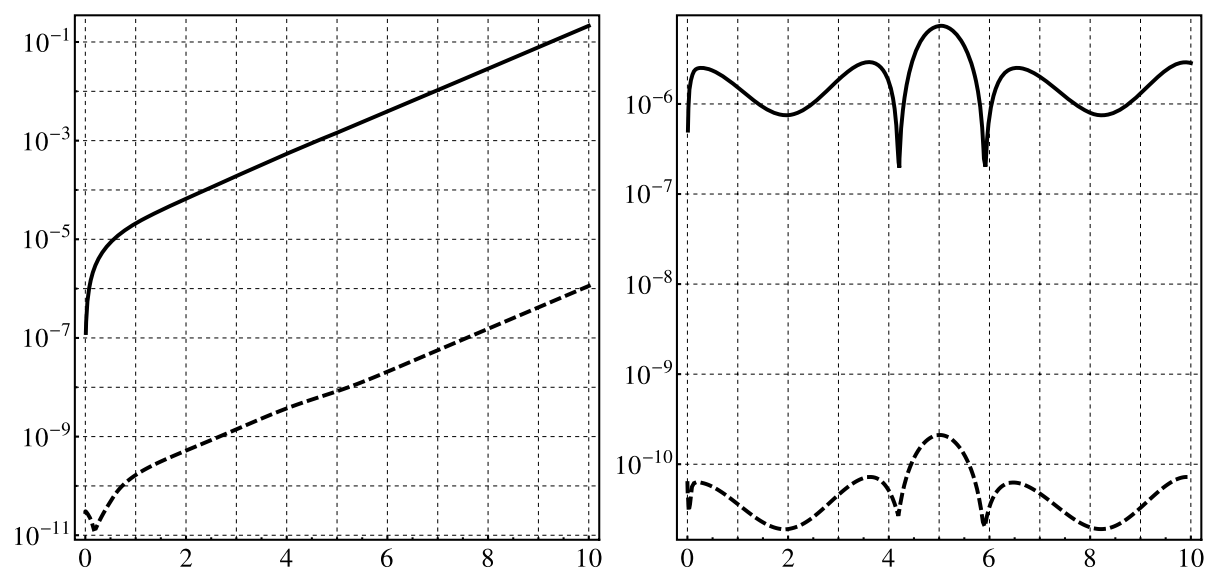

Fig. 5. The error (in log scale) of the solution of the difference scheme (7)-(10) vs time $t$, provided that the exact solution is defined by (24). $\alpha(x)=x, \beta(x)=\cos x, T=10, N=200, h=0.005$. Solid curve represents the case $\tau=0.01(\sigma=599 / 1200 \approx 0.499)$, dashed curve - the case $\tau=0.00005(\sigma=1 / 3)$. Figure on the left: $\gamma_{1}=e /(e-2) \approx 3.78442, \gamma_{2}=2 /(e+\sin 1-\cos 1) \approx 0.662372$. Figure on the right: $\gamma_{1}=-4, \gamma_{2}=8$. 

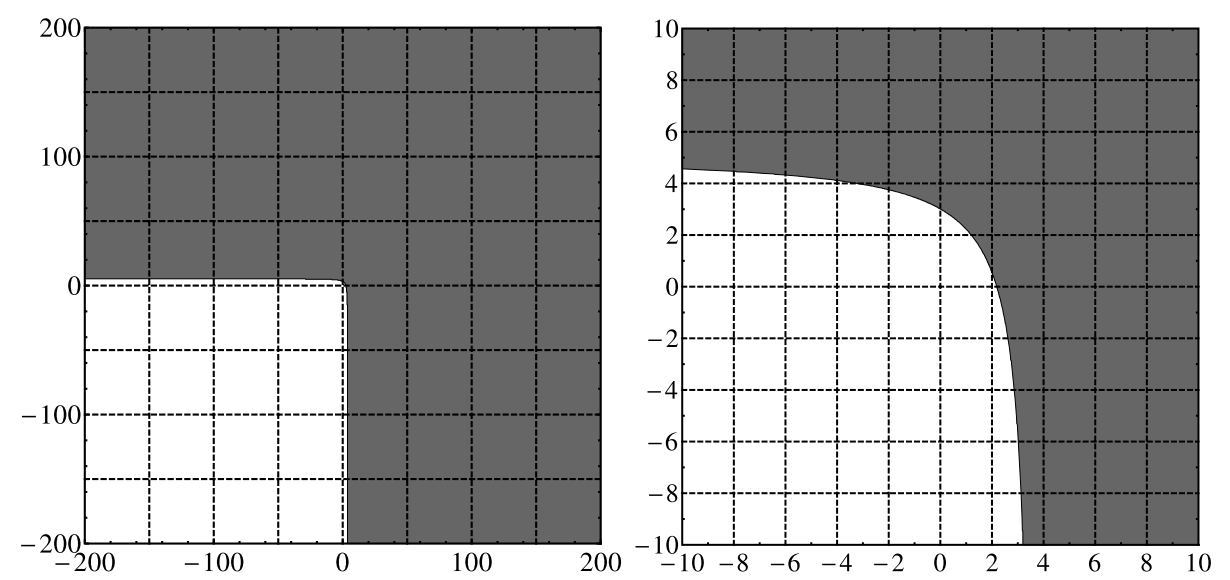

Fig. 6. Properties of the spectrum of the matrix $A$ in the plane $\left(\gamma_{1}, \gamma_{2}\right) . \alpha(x)=\cos x, \beta(x)=x, N=200$, $h=0.005$. Figure on the right presents the zoom of the figure on the left.

The stability of the difference scheme essentially depends on the functions $\alpha(x), \beta(x)$. Let's compare two problems - one for $\alpha(x), \beta(x)$ defined by the functions (23), and another one with the swapped functions

$$
\alpha(x)=\cos x, \quad \beta(x)=x .
$$

The regions of stability and instability of the later problem (when $\alpha(x)$ and $\beta(x)$ are chosen by the formulae (25)) are presented in Fig. 6 (the same as in Figs. 3 and 4 graphical notations of different background regions are employed). Note that in this case the matrix $A$ has no complex (with non-zero imaginary part) eigenvalues.

Therefore, if the point $\left(\gamma_{1}, \gamma_{2}\right)$ is lower to both branches of the hyperbola (20) (belongs to the region of white background, see Fig. 6), all eigenvalues of the matrix $A$ are positive and the difference scheme is stable.

But, if the point $\left(\gamma_{1}, \gamma_{2}\right)$ is between branches of the hyperbola (the matrix $A$ possesses one negative eigenvalue) or above both branches of the hyperbola (two negative eigenvalues of $A$ appear), the difference scheme is unstable - since at least one negative eigenvalue exists. In Fig. 6 such points $\left(\gamma_{1}, \gamma_{2}\right)$ are located in the region of gray background.

By comparing Figs. 4 and 6, we conclude, that the values of $\left(\gamma_{1}, \gamma_{2}\right)$ for which the difference scheme is stable, essentially depend on the order ((23) or (25)), in which the functions $\alpha(x)$ and $\beta(x)$ are defined.

Test 3. In this test we choose (in the problem (1)-(4))

$$
\alpha(x)=1+x, \quad \beta(x)=0 .
$$


Then, the characteristic curve (20) defines a line, parallel to the axis $\gamma_{2}$ :

$$
\gamma_{1}=\frac{6}{5}
$$

Therefore, the regions of stability and instability are defined quite simply:

- the matrix $A$ possesses one negative eigenvalue and the scheme is unstable if $\gamma_{1}>6 / 5$

- the matrix $A$ has no eigenvalues with the property $\operatorname{Re} \lambda_{i}(A)<0$ and the scheme is stable, if $\gamma_{1}<6 / 5$. More precisely, all eigenvalues of $A$ are positive, when $-a<\gamma_{1}<6 / 5, a \approx 2.8$; complex eigenvalues (with non-zero imaginary part), holding the property $\operatorname{Re} \lambda_{i}(A)>0$ exist, when $\gamma_{1}<-a$.

Test 4. In the paper of Martin-Vaquero and Vigo-Aguiar (2009a) the example from the paper Day (1982) has been provided, $\alpha(x)$ and $\beta(x)$ being chosen as

$$
\alpha(x)=-2 \delta^{2}(2-3 x), \quad \beta(x)=2 \delta^{2}(1-3 x) .
$$

In this case the problem (1)-(4) portrays the quasi-static flexure of a thermoelastic rod unit length. We consider this example by applying our method (subject to stability of difference scheme) for the functions

$$
\alpha(x)=2-3 x, \quad \beta(x)=1-3 x .
$$

For these $\alpha(x)$ and $\beta(x)$, the characteristic curve (20) degenerates into two lines (parallel to the coordinate axes $\left.\gamma_{1}, \gamma_{2}\right)$, crossing each other at the point $\left(\gamma_{1}=2, \gamma_{2}=-2\right)$ (see Fig. 7).
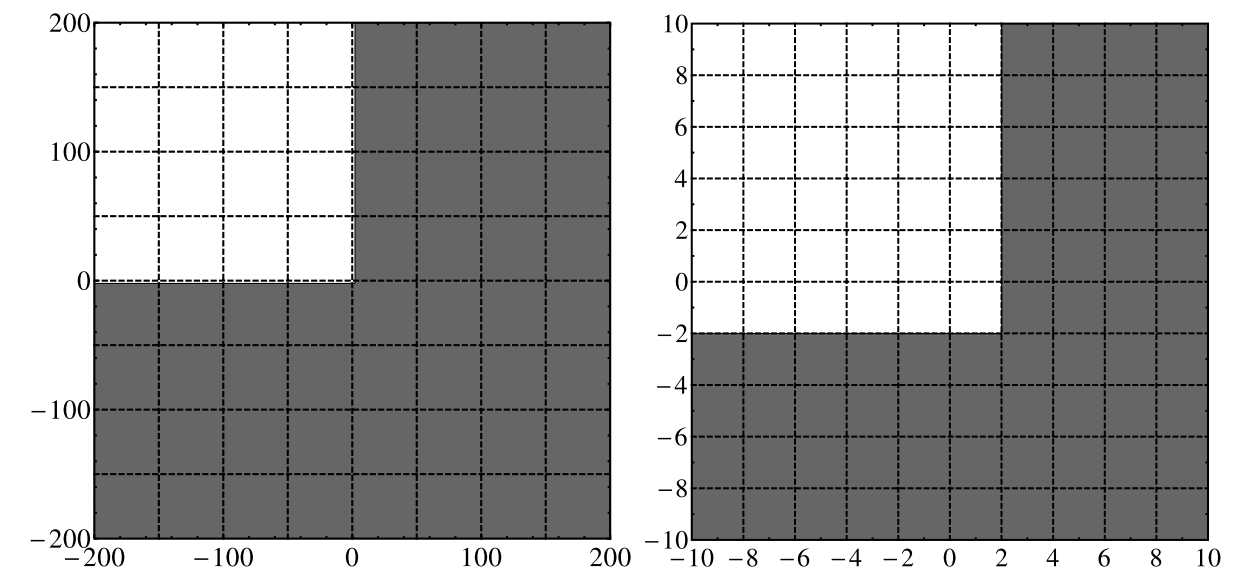

Fig. 7. Properties of the spectrum of the matrix $A$ in the plane $\left(\gamma_{1}, \gamma_{2}\right) . \alpha(x)=2-3 x, \beta(x)=1-3 x$, $N=200, h=0.005$. Figure on the right presents the zoom of the figure on the left. 
The matrix $A$ has no complex (with non-zero imaginary part) eigenvalues. Hence, in the entire region $\left\{\gamma_{1} \leqslant 2, \gamma_{2} \geqslant-2\right\}$ of $\left(\gamma_{1}, \gamma_{2}\right)$ coordinate plane the difference scheme is stable, but unstable in the rest of the coordinate plane.

Also, it should be noted that, if $\alpha(x)$ and $\beta(x)$ are defined by the expressions (26), the difference scheme is stable for all real values of $\delta$.

\section{Conclusions and Generalizations}

In this study we propose a systematic and generalized methodology, how to investigate the influence of the weight functions $\alpha(x)$ and $\beta(x)$ (present in the integral boundary conditions), on the stability of difference schemes. The ground of the methodology is the investigation of the spectrum of a matrix, defining the transition to the upper layer of the difference scheme. Main conclusions concerning the stability of the difference scheme with nonlocal conditions are reached by complimenting the methodology mentioned with computational experiment.

All conclusions of Section 3 are reached by performing extensive computational experiment. In this paper we present only small part of it. During computational analysis all eigenvalues of the matrix $A$ were examined with different values of the grid step $h$ (but most often with the values $h=1 / 200$ or $h=1 / 100$ ). The eigenvalues for different values of $\gamma_{1}, \gamma_{2}$ were calculated in the interval $-200 \leqslant \gamma_{1}, \gamma_{2} \leqslant 200$. Generalizing the results of Section 3 we could say that the stability of difference schemes essentially and complicatedly depends on the weight functions $\alpha(x)$ and $\beta(x)$.

Although we reached these conclusions by analysing spectrum of the matrix of the difference operator, it is not the only method to investigate the stability. In many cases the information about the stability of a difference scheme can be obtained from study of the characteristic curve (20), expression of which is quite simple. We observed from computational experiment that the zone of stability either coincides with the domain $S_{1}$ (see Fig. 1) or it is part of the domain $S_{1}$ (in the case when part of the eigenvalues of the matrix $A$ are complex, with non-zero imaginary part). Neither in the results of computational experiment, presented in this paper, nor in other cases we have observed the zone of stability would extend over the domain $S_{1}$.

Investigating the problem of stability we were about to get at least partial answer to the question: if the quite simple scheme (7)-(10) with $\sigma=1$ and the functions $\alpha(x)$ and $\beta(x)$ is unstable, is it possible to build a stable difference scheme, by partially or essentially modifying the (7)? Yet we have no positive answer. The indirect positive answers of some other authors also do not persuade us. The question is still open.

\section{References}

Cannon, J.R. (1963). The solution of the heat equation subject to specification of energy. Quarterly Applied Mathematics, 21(2), 155-160.

Čiegis, R., Tumanova, N. (2010). Numerical solution of parabolic problem with nonlocal boundary conditions. Numerical Functional Analysis and Optimizaton, 31(12), 1318-1329.

Čiegis, R., Štikonas, A., Štikonienè, O., Suboč, O. (2002). A monotonic finite-difference scheme for a parabolic problem with nonlocal conditions. Differential Equations, 38(7), 1027-1037. 
Čiupaila, R., Jesevičiūtė, Ž., Sapagovas, M. (2004). Eigenvalue problem for ordinary differential operator subject to integer condition. Nonlinear Analysis: Modelling and Control, 9(2), 109-116.

Day, W.A. (1982). Extensions of a property of the heat equation to linear thermoelasticity and order theories. Quarterly Applied Mathematics, 40, 319-330.

Dehghan, M. (2005). Efficient techniques for the second-orfer parabolic equation subject to nonlocal specifications. Applied Numerical Mathematics, 52(1), 39-62.

Dehghan, M. (2007). The one-dimensional heat equation subject to a boundary integral specification. Chaos, Solitons and Fractals, 32(2), 661-675.

Ekolin, G. (1991). Finite difference methods for a nonlocal boundary value problem for the heat equation. BIT, $31,245-261$

Gao, J., Sun, D., Zhang, M. (2010). Structure of eigenvalues of multi-point boundary value problems. Advances in Difference Equations.

Gulin, A., Ionkin, N., Morozova, V. (2006). Stability criterion of difference schemes for the heat conduction equation with nonlocal boundary conditions. Computational Methods in Applied Mathematics, 6(1), 31-55.

Ivanauskas, F., Meškauskas, T., Sapagovas, M. (2009). Stability of difference schemes for two-dimensional parabolic equations with nonlocal boundary conditions. Applied Mathematics and Computation, 215(7), 2716-2732.

Jachimavičienè, J., Jesevičiūte, Ž., Sapagovas, M. (2009). The stability of finite-difference schemes for a pseudoparabolic equation with nonlocal conditions. Numerical Functional Analysis and Optimization, 30(9), 988-1001.

Jesevičiūtè, Ž., Sapagovas, M. (2008). On the stability of the finite-difference schemes for parabolic equations subject to integral conditions with applications for thermoelasticity. Computational Methods in Applied Mathematics, 8(4), 360-373.

Kalna, G., McKee, S. (2004). The termostat problem with a nonlocal nonlinear boundary condition. IMA Journal of Applied Mathematics, 69, 437-462.

Lin, Y., Xu, S., Yin H.M. (1997). Finite difference approximations for a class of non-local parabolic equations, International Journal of Mathematics and Mathematical Sciences, 20, 147-163.

Martin-Vaquero, J., Vigo-Aguiar, J. (2009a). A note on efficient techniques for the second-order parabolic equation subject to non-local conditions. Applied Numerical Mathematics, 59, 1258-1264.

Martin-Vaquero, J., Vigo-Aguiar, J. (2009b). On the numerical solution of the heat conduction equations subject to nonlocal conditions. Applied Numerical Mathematics, 59, 2507-2514.

Medvedev, V., Dzemyda, G. (2006). Optimization of the local search in the training for SAMANN neural network. Journal of Global Optimization, 35, 607-623.

Medvedev, V., Dzemyda, G., Kurasova O. (2011). Efficient data projection for visual analysis of large data sets using neural networks. Informatica, 22(4), 507-520.

Nakhushev, A.M. (1995). The Equations of Mathematical Biology. Nauka, Moscow (in Russian).

Samarskii, A.A. (1979). Mathematical modeling and computational experiment. Vestnik Akademii Nauk SSSR, 5, 38-49 (in Russian).

Samarskii, A.A. (2001). The Theory of Difference Schemes. Marcel Dekker, New York.

Sapagovas, M. (2008). On the stability of a finite-difference scheme for nonlocal parabolic boundary-value problems. Lithuanian Mathematical Journal, 48(3), 339-356.

Sapagovas, M., Štikonas, A. (2005). On the structure of the spectrum of a differential operator with a nonlocal condition. Differential Equations, 41(7), 1010-1018.

Sapagovas, M., Štikoniene, O. (2011). Alternating-direction method for a mildly nonlinear elliptic equation with nonlocal integral conditions. Nonlinear Analysis: Modelling and Control, 16(2), 220-230.

Schuegerl, K. (1987). Bioreaction Engineering. Reactions Involving Microorganisms and Cells, Vol. 1. Wiley, New York.

Skučaitè, A., Skučaitė-Bingelè, K., Pečiulyte, S., Štikonas, A. (2010). Investigation of the spectrum for the Sturm-Liouville problem with one integral boundary condition. Nonlinear Analysis: Modelling and Control, $15(4), 501-512$.

Štikonas, A. (2011). Investigation of characteristic curve for Sturm-Liouville problem with nonlocal boundary conditions on torus. Mathematical Modelling and Analysis, 16(1), 1-22.

Yin, H.M. (2004). On a class of parabolic equations with nonlocal boundary conditions. Journal of Mathematical Analysis and Applications, 294, 712-728. 
M. Sapagovas graduated from the Vilnius University in 1961. He received the doctoral degree in mathematics (PhD) at the Institute of Mathematics in Kiev in 1965. In 1986 he received the doctor habilitatus degree from the M. Keldysh Institute of Applied Mathematics in Moscow. M. Sapagovas is a professor (1989), a member of the Lithuanian Academy of Sciences (1987), a principal researcher of the Department of Numerical Methods at the Institute of Mathematics and Informatics of Vilnius University (2005). The main field of scientific interest is the numerical methods for nonlinear PDE as well as mathematical modeling.

R. Čiupaila has graduated Vilnius University Mathematics Faculty in 1979. He received a doctoral degree in 1992 in Minsk (Belorus Republic). He received scientific title of an associated professor of Vilnius Gediminas technical university in 2002. The main field of research is a solution of PDE with nonlocal conditions.

Ž. Jokšienė (Jesevičiūtè) graduated from the Informatics Faculty of the Vytautas Magnus University, Kaunas in 2005 as mathematics program master. She received doctor degree in the Institute of Mathematics and Informatics in 2010 in mathematics. She is lecturer at the Department of Physics, Mathematics and Biophysics at Lithuanian University of Health Sciences, Medical Academy. Also she is working in Vytautas Magnus University as lecturer. Research interests: numerical methods, the differential equations with nonlocal conditions, structure of spectrum for the differential operator with nonlocal integral conditions.

T. Meškauskas graduated from Faculty of Mathematics and Informatics, Vilnius University, in 1994. In 1999 he earned doctoral degree (in mathematics), also from Faculty of Mathematics and Informatics, Vilnius University. Two years (2000-2002) spent doing postdoctoral studies at CERFACS (Toulouse, France). As of 2012, he is associate professor at Faculty of Mathematics and Informatics, Vilnius University. Research interests: numerical methods for partial differential models, mathematical and numerical modeling of electrochemical phenomena in tethered bilayer membranes, chaos theory, $1 / f$ noise.

\section{Skaičiavimo eksperimentas skirtuminiu schemu su nelokaliosiomis salygomis stabilumui tirti}

Mifodijus SAPAGOVAS, Regimantas ČIUPAILA, Živilè JOKŠIENĖ, Tadas MEŠKAUSKAS

Straipsnyje aprašyta metodika, kaip gauti naujus diferencialinių lygčiu skaitinių algoritmu rezultatus, naudojant skaičiavimo eksperimentą kaip tyrimo metodą. Pateikti nauji rezultatai apie parabolinių lygčių su nelokaliosiomis integralinėmis sąlygomis skirtuminių schemų stabilumą. 